\title{
Piglia escritor
}

\section{(4) Alejandra Laera}

Instituto de Literatura Hispanoamericana, Facultad de Filosofía y Letras, Universidad de Buenos Aires / CONICET, Argentina

Recibido: 14/4/2018. Aprobado: 6/6/2018.

\begin{abstract}
Resumen
El artículo plantea que, en Los diarios de Emilio Renzi, Ricardo Piglia describe y define la condición del escritor a partir de la postulación de un hiato entre vida y literatura que elabora esta relación de un modo diferente a como lo había hecho en su obra previa. Asimismo, el artículo analiza la relación entre el tiempo de la escritura de los diarios y el tiempo de la edición de Los diarios, y propone que el editor, por medio de procedimientos de ficcionalización tales como el desdoblamiento autoral y la manipulación temporal, conspira contra el escritor y su relato de vida redefiniendo su condición futura al borde de la muerte.
\end{abstract}

\section{Piglia, writer}

\begin{abstract}
This essay poses that Ricardo Piglia's Los diarios de Emilio Renzi, describes and defines the condition of being a writer, based on a sort of hiatus between life and literature. Also, the essay analizes the connection between the journal's writing, and its publishing as a book, and holds that the editor (Piglia) conspires through fictionality not only against time, but also against the writer (Piglia/Renzi) and the narration of his life, redifining his figure in the threshold of death.
\end{abstract}

Keywords

Ricardo Piglia Writer Journal Life Fiction Temporality

'Bueno, voy a ser escritor', le dije, tenía dieciséis años y tantas posibilidades de ser escritor como de ser aviador o mercenario. Vivir la literatura como un destino no garantiza la calidad de los textos, pero asegura la convicción necesaria para elegir en cada momento. Uno vive una vida de escritor porque ya lo ha decidido, pero luego los textos deben estar a la altura de esa decisión. 
1. Para un acercamiento general de corte sociológico a algunas de estas cuestiones, ver Nathalie Heinich (2000), quien, entre otras reflexiones, propone la distinción entre autopercepción, representación y designación para pensar el "ser escritor", que de hecho le da título a uno de sus libros. También, y reformulando el abordaje señero de Pierre Bourdieu, ver Bernard Lahire (2006) y su explicación sobre la "doble vida de los escritores".
¿Qué es "ser escritor"? ¿Ser escritor es sentirse escritor o es más bien el deseo de serlo? ¿Será la vocación? Ser escritor: ¿es estrictamente la actividad de ponerse a escribir? ¿O es en cambio publicar? ¿O mejor aún: es ser reconocido como tal? Y ser reconocido como tal: ¿por los pares o por un público? ¿Y cuándo: ahora o de manera póstuma? ¿O ser escritor es ser un escritor profesional que puede vivir de una escritura consensuadamente identificada como literaria? ¿Es acaso trabajar de escritor, cobrar por lo que se escribe? Ser escritor, en definitiva, ¿qué es? ¿Una autopercepción, un proyecto, una actividad, un resultado, un trabajo? Y desde otro punto de vista: ¿se trata de un proceso o es una conversión que se da de un momento para el otro?

Estas preguntas, que obviamente tienen más de una respuesta a lo largo del tiempo y también varias a la vez, resuenan en la Argentina con insistencia en ciertas condiciones: pueden ser aquellas que permitieron soñar con la ocupación de literato en los tiempos del Romanticismo (Esteban Echeverría y la pionera publicación de un par de volúmenes de poemas entre 1832 y su exilio montevideano a finales de la década es el ejemplo más nítido); pueden ser las que les pusieron drama a los años de 1930 cuando ya era reconocible una industria cultural (como fue el caso de Roberto Arlt, debatido entre el deseo de escribir novelas y el trabajo periodístico), o pueden ser, entre otras, las condiciones que habilitan un presente de proliferación de escritores y escritoras, un presente con altas chances de que un escritor sea capaz, antes que nada, de visibilizarse como tal (basta atender, a modo de muestra, a los relatos que se adelantan en los posteos de Facebook y después forman un volumen, como ocurrió con las crónicas que forman Piletas [2017] de Félix Bruzzone). Se trata de preguntas insistentes cuyas formulaciones y respuestas oscilan entre la mirada de los investigadores (estudiosos de la literatura, sociólogos de la cultura), la construcción de los propios escritorxs (en textos autobiográficos, entrevistas, relatos documentales) y, sobre todo, el montaje que de ambos gestos consigue hacer la crítica cultural. ${ }^{1}$

La escena de la conversión en escritor -que no debe confundirse, según puede inferirse de lo que planteé hasta acá, con la escena de iniciación a la escritura- ha sido privilegiada en la mayoría de los relatos aubiográficos de escritores y escritoras argentinos/ as, aunque no con pareja eficacia. Muy probablemente, una de las primeras y más potentes escenas de ese "ser escritor" la protagoniza Domingo Faustino Sarmiento y está en Recuerdos de provincia (la narra en 1850 y la data en 1841). Allí Sarmiento afirma dos veces "yo era escritor": primero, cuando le cuentan el gesto aprobatorio de uno de sus maestros al leer su primer artículo publicado en la prensa y, después, tras escuchar semiescondido en una reunión las expresiones alabatorias con las que un grupo de letrados ha recibido ese mismo artículo. "Yo era escritor, pues." (Sarmiento, 1979: 168). En su caso, y como se observa en el armado de ambas escenas, la declaración no remite al momento en que está llevando a cabo la tarea de escribir (que por otra parte no aparece nunca en Recuerdos de provincia), tampoco al hecho en sí de la primera publicación. En cambio, Sarmiento "es escritor" (se siente escritor, se convierte en escritor, se considera escritor) cuando los "hombres notables" lo leen y lo reconocen como escritor: la autopercepción depende del reconocimiento de un grupo de lectores distinguidos. Casi en el otro extremo de la inmodestia sarmientina, un siglo después, Jorge Luis Borges hace de su naturaleza de lector su explícita condición de escritor: se ratifica, en entrevistas y ensayos, lector antes que escritor; borra las huellas de la escritura en su dimensión material pero también en su dimensión simbólica; se desprende de designaciones y autodesignaciones, se desprende del trazo... (Borges, 2005). Ser escritor es su precuela: ser lector. En el medio de ambos extremos, desde ya, no solamente una construcción que resulta o bien más impúdica o bien más modesta del escritor como las que mencioné, sino incluso la captación casi sociológica de un momento propicio como lo fueron las primeras décadas del siglo XX para pensar allí indiscutiblemente a los escritores como un grupo, como una suerte de gremio; un momento en el que ser escritor era algo dado, de lo que se podía formar 
parte aun cuando se pusiera en juego la legitimidad literaria de ese nuevo modo de serlo. Sin dudas, Roberto J. Payró es el caso testigo: la correlación entre sus crónicas periodísticas de comienzos de siglo (que describen la actividad y buscan regularla legalmente), varios de sus relatos (como el dilemático "La paradoja del talento") y su obra de teatro El triunfo de los otros ilustra inmejorablemente el modo en que los escritores se han reposicionado en el campo social (Payró, 1984 y 1907). Por supuesto, se trata solo de algunos ejemplos, circunstancias, situaciones, que podrían ampliarse con otros para considerar matices muy diversos de ese juego entre lo documental y la construcción filoficcional que supone la posibilidad de serescritor.

\section{El escritor y sus vidas}

Dentro de un conjunto muy variado, los tres volúmenes que integran Los diarios de Emilio Renzi de Ricardo Piglia se imponen como uno de los materiales más valiosos para repensar las preguntas que planteé al comienzo. En ellos, Piglia no solo da una respuesta personal a la pregunta sobre "qué es ser escritor", sino que parece responder a cada una de sus posibilidades, de sus variaciones, a la vez que, por esa vía, el diario se convierte en la poética del escritor y en su puesta en relato en primera persona. Con los diarios, la vida, la escritura y la literatura se traman de un modo que avanza irremisiblemente en la propia relación que Piglia había tejido en su obra entre experiencia y literatura. Con los diarios, la vida se convierte en obra, y en esa conversión, lo fundamental es que se trata de una vida concebida siempre en tanto vida de escritor.

En Los diarios de Emilio Renzi, la pregunta "qué es ser escritor" se plantea desde el vamos, reiterada, obsesivamente. Piglia presenta y discute cada definición, la asume, la transforma, la desecha. Lo hace de entrada, ya en el período de los diarios correspondiente al bienio 1959-1960, todavía adolescente: "Cuando releo lo que tengo escrito de la monografía me quiero morir. ¿De dónde saqué que soy escritor?" (2015:48). Y también cuando está terminando su primer libro y piensa entonces en los lectores, en los críticos: "Ahora, con el libro casi listo, veo venir el encuentro real con la literatura, casi diez años después de haber hecho la apuesta a todo o nada. ¿Seré capaz de superar un fracaso?" (2015: 212). Pero es justamente en esa misma instancia cuando, en el revés del posible fracaso, se rediseña un perfil más depurado de escritor, más pretendidamente incontaminado de trabajos que, desde la concepción de la literatura que busca por entonces sostener Piglia, serían no genuinos sino subsidiarios: "Todavía no conozco mis límites, tengo que empezar a delimitar el espacio de mi vida. Por momentos tengo la imagen de ser una máquina que sirve para todas las funciones. Y, sin embargo, la única máquina que conozco de cerca es la Olivetti en la que escribo, es allí donde debo probar mi alcance." (2015: 298) Así, la condición de escritor depende, según las circunstancias y la situación personal, de la vocación, de la escritura literaria propiamente dicha, de la publicación, del valor, de la distinción respecto de otros trabajos. Piglia termina poniendo en duda todas las definiciones, a la vez que por momentos las afirma. Y todo eso lo hace en el juego que entabla entre experiencia y escritura (entre la experiencia de la escritura y la escritura de la experiencia, por proponer un juego de palabras en sintonía con el que proponen los mismos diarios).

Atribuidos a esa suerte de alter ego del escritor que hasta protagonizó algunas de sus novelas como Respiración artificial (1980) o El camino de Ida (2013), Los diarios de Emilio Renzi fue el modo público que asumió el diario personal que Ricardo Piglia había llevado adelante a lo largo de más de cincuenta años en muchísimos y sucesivos cuadernos, en paralelo a su producción ensayística y ficcional. Es una obra compuesta por tres volúmenes publicados en 2015, 2016 y 2017: Años de formación, que abarca el período entre 1957 y 1967; Los añosfelices, que va de 1968 a 1975, y Un día en la vida, que 
2. Sobre los problemas generales de la autobiografía desde perspectivas históricas, teóricas y filosóficas, siguen siendo muy vigentes los estudios clásicos de Gusdorf (1991), De Man (1979) y Starobinski (1970), además del discutido abordaje específico de Lejeune (1975), que fue dominante en las teorizaciones sobre el género hasta los años 90 e introdujo la noción de "pacto".

3. Si bien podria decirse que el párrafo corresponde no a la fecha del enunciado sino a la edición del diario, hay que recordar, de todos modos, que la importancia de la relación entre el Yo y el Otro en el pensamiento de Piglia puede datarse fehacientemente hacia la década de 1990, cuando se conoce su breve pero seminal ensayo "Echeverría y el lugar de la ficción". Allí, da una de las claves para pensar la literatura argentina del siglo XIX: declara allí que mientras la clase se cuenta a sí misma con la forma de la autobiografía, el otro es contado a través de la ficción (Piglia, 1993). toma entre 1976 y 1982 y recorre el resto de los años hasta poco antes de la muerte en 2017 , sin datación precisa. Los tres volúmenes transcriben el contenido de unos trescientos cincuenta y siete cuadernos autobiográficos, que es sometido a un expreso proceso de edición. A ese contenido: lo resumen, condensan, acortan; lo modifican, corrigen, sustituyen; lo expanden, lo intersecan, lo intervienen. Piglia resulta ser ahora, además de escritor, editor de sí mismo.

De lo que se trata, entre otras cosas pero privilegiadamente, es de narrar en los cuadernos y editar en los diarios lo que, en una reseña publicada tras la salida del segundo volumen, Alan Pauls (2016) llamó, a partir de una expresión del propio Piglia, "la Forma de Vida Escritor", que incluye

a los escritores que se admira, los problemas formales o de estilo que desvelan, las ideas sobre la literatura que se quiere poner a prueba, pero también ardides para administrar el dinero y las comidas, formas de gestionar la sociabilidad, las pasiones y el afecto, modos de optimizar el aprovechamiento del tiempo: todo un arte de la economía y la autogestión vital puesto al servicio de una causa superior: la devoción a la escritura. (Pauls, 2016)

Se trata, en definitiva, de vivir el pasaje que va de sentirse escritor a decidir hacerse tal y a finalmente serlo. Pasaje realizado a través de una Forma de Vida pero, en igual medida y al mismo tiempo, propongo, de una Escritura, que nos conduce a la pregunta que planteé al comienzo, la misma con la que casi se inicia el diario, o mejor dicho, la edición del diario:

¿Cómo se convierte alguien en escritor, o es convertido en escritor? No es una vocación, a quién se le ocurre, no es una decisión tampoco, se parece más bien a una manía, un hábito, una adicción, si uno deja de hacerlo se siente peor, pero tener que hacerlo es ridículo, y al final se convierte en un modo de vivir (como cualquier otro). (Piglia, 2015: 16)

El fragmento plantea ya la preocupación principal del diario; lo hace retrospectivamente, ya que la definición está incluida en esa suerte de prólogo al primer volumen llamado "En el umbral", en el que Renzi aparece como personaje conversando con quien se supone es el autor, Piglia, y lo hace anticipando de qué se va a hablar, porque el "umbral" es el umbral de los diarios de vida y también de la conversión en escritor. Pero además, plantea la principal preocupación de su protagonista en el mismo momento en el que el Yo del escritor se desdobla en Renzi. El Yo, de los diarios, de la autobiografía, rompe el pacto y propone un modo de mirarse a sí mismo como escritor desde su Otro: Yo soy el Otro que me mira como si Yo fuera Otro. Dicho de otro modo: para poder ser Yo tengo que ser Otro. Ser escritor, entonces, es también ser Otro sin dejar de ser Yo mismo. ${ }^{2}$ Todo eso así, como para empezar, puesto allí justo antes del registro del primer año, en 1957, cuando todavía era un adolescente. Pero hay algo en esto que quiero destacar: más allá de las explicaciones dadas por Piglia o por Piglia/Renzi, en estos desdoblamientos se pone de manifiesto la relación inescindible entre vida y literatura. Un párrafo de la entrada del jueves 10 de febrero de 1966, ¿no alude acaso a esto mismo?: "Por mi lado, como siempre cuando estoy en peligro, quisiera escribir sobre mí en tercera persona. Evitar la ilusión de 'tener' una vida interior." (2015: 228). ${ }^{3}$ Es entonces en esa doble posición enunciativa (Piglia/Renzi), en ese umbral, también, que habilita una ficcionalización, desde donde se habla del "ser escritor" a la vez como escritor (el autor Piglia y el escritor Renzi, el yo y el otro) y como lector (Piglia que lee a Renzi, el yo que se lee a sí mismo como si leyera a otro).

Volvamos entonces ahora a las preguntas de apertura formuladas en el volumen inicial, Años de formación. Allí mismo aparecen algunas respuestas tentativas que se irán 
confirmando o rectificando. La primera, de hecho, está en el mismo prólogo, "En el umbral", al referirse a las causas por las que alguien se dedica a escribir y al reemplazar su importancia por las consecuencias; se dice entonces: "Un escritor se autodesigna, se autopropone en el mercado persa" (2015: 18), lo que supone autodesignarse solo cuando se tiene una obra, de allí la autopostulación en el mercado (como si dijéramos: ya tengo la obra, a ver ahora qué pasa, de qué modo circulo como escritor). Sin embargo, apenas un poco después, pero ya en una entrada temprana, o sea como registro previo al prólogo que enmarca los diarios y que corresponde explícitamente a la edición, Piglia, tras narrar un encuentro con Borges, hace una declaración que parece acercarlo a Sarmiento aun cambiándole por completo el sentido: "En fin, me hundió, pero me reconoció como escritor, ¿no es cierto?, dijo Renzi [...] en fin, las ilusiones tienen que ser confirmadas alguna vez por otro, aunque sea por medio de la humillación y el espanto" (2015: 31). Estoy tratando de ilustrar el despliegue de posibilidades y de definiciones sobre el "ser escritor" que va ofreciendo Piglia a través de Renzi y por medio de la composición de los diarios, de un modo que, por eso mismo, porque es tan diferente de una novela o un cuento como de un ensayo, no resulta ni progresivo ni homogéneo; más todavía: no aparece nunca acabado o cristalizado, no resulta nunca secuencialmente coherente con una lógica ficcional ni fundamentado en una lógica argumentativa. En cambio, sigue los vaivenes de la primera persona autobiográfica, de sus circunstancias, su estado de ánimo, su variabilidad. Y eso no deja de ocurrir nunca, como cuando el último día de 1977 Piglia/Renzi dice, refiriéndose a Respiración artificial, cuya publicación tardaría tres años, que "Quizás alguna vez pueda leer publicada esta novela que ahora me da tanto trabajo escribir." (2016: 62). Porque en ese "ser escritor" siempre hay un drama, algo del orden de lo irresoluble: la escritura que todavía no es y que tiene que venir, la inminencia muchas veces pospuesta de la escritura, las dudas sobre su valor, los miedos ante las futuras lecturas (de los pares, de la crítica, del público)... Una escritura que, para que nazca, surja, emerja, se forme el Escritor (y no solo su Forma de Vida), tiene que estar reunida en un volumen.

Porque finalmente, ¿qué es "ser escritor"? Casi terminando lo que llama "años de formación", en 1967, Piglia/Renzi se entera el mismo día de que le van a publicar su primer libro de cuentos, La invasión, y de que ese libro de cuentos, prácticamente igual y con el nombre de Jaulario, ganó la primera mención del premio Casa de las Américas y será también publicado en Cuba (2015: 290). Pero no solo eso, si consideramos el estado posterior y actual del mercado del libro en la Argentina y en América Latina en general, bastante reducido respecto de la etapa gloriosa que atravesó entre mediados de los años 50 y comienzos de los 70: se habla entonces de ediciones de miles de ejemplares (¡¿diez mil ejemplares?!), cifra que al Piglia lector de sus diarios, ya consagrado, traducido a varios idiomas y con amplia circulación editorial y mediática en lengua española, no habrá podido dejar de asombrarle como ingreso en el mundo editorial (¿o no es por los números que se mide el éxito de la autopostulación en el "mercado persa"?). Allí entonces termina el volumen dedicado a los años de formación: al publicar el primer libro. En el siguiente volumen, Piglia ya es escritor, aunque los dramas de la Escritura y de la Forma de Vida Escritor, como pudo verse, continúan hasta el final.

\section{El editor y sus conspiraciones}

Es cierto, como dice Pauls en la reseña que ya mencioné, que mientras el proceso, lo paulatino, se desenvuelve como Forma de Vida Escritor, al escribir un diario la identidad escritor se asume de manera fulminante y es del orden de lo performativo: escribir un diario es declararse escritor (Pauls, 2016). Ahora bien: ese escritor, ¿es el mismo que se convierte en tal en el relato del diario? Digamos: ¿el escritor enunciado en el diario es el mismo que lo enuncia? 
Si la relación Piglia/Renzi planteada desde el vamos apunta a una diferencia de orden textual en cuyo desdoblamiento se habilita la ficcionalización, hay también en los diarios un señalamiento de otro orden, que excede los recursos de corte literario, como al escribir, a fines de 1977, que "También este cuaderno sería leído, alguna vez en el futuro, por alguien que no será este que soy ahora" (Piglia, 2016: 62). Interpretada como parte de la edición de los diarios, la frase se verifica casi lúdicamente en el mismo procedimiento narrativo del desdoblamiento: en efecto, Piglia hará que Renzi lea a Piglia que a su vez leerá a Renzi. Leída en cambio como frase genuina de los diarios, lo que prevé es que la experiencia (cabe inferir: de vida y de escritura) hará de quien escribe los diarios alguien diferente. Pero, dando un paso más, si se la considera sin atarla a su valor de verdad, permite entender el desdoblamiento no solo como un juego narrativo sino como el recurso que pone en evidencia desde el vamos la transformación del protagonista de los diarios a lo largo del tiempo. El procedimiento Piglia/ Renzi, así, es multivalente: es a la vez un guiño ficcional, un indicio extratextual, una resolución narrativa para un conflicto personal, un modo de vincular las condiciones de enunciación del pasado con las del presente, un modo, también, de distinguirlas. Esa distancia entre ambos escritores, instalada por la transformación inherente a la experiencia acumulada a lo largo de los años y marcada por el desdoblamiento de Piglia en Renzi, se corrobora, potenciada, cuando el escritor de Los diarios de Renzi se convierte en el editor de los diarios de Piglia. Porque el editor de los diarios es el suplemento del escritor de los cuadernos. Quiero decir: la operación que realiza el autor Ricardo Piglia consiste tanto en el desdoblamiento del escritor Piglia en el escritor Renzi como en la constitución del autor Piglia en editor de sí mismo. ¿Pero no es en ese punto, justamente, allí donde se construye el artificio y se advierte una potencial ficción, donde la literatura se revincula con la vida?

Casi literalmente, se puede asumir que la elección de la autobiografía como forma narrativa no solo supone esa relación sino que la enfatiza: la autobiografía, sobre todo si se amplía el ámbito de la pura escritura y se la da a conocer por medio de la publicación, enlaza la vida con la literatura. Pero que se trate de diarios, con su cronología detallada, con su escritura de tiempo presente, de recuerdo o de memoria inmediata, no implica únicamente un retorno al pasado, como otras formas de la autobiografía, sino su actualización. Y es en esa actualización del tiempo donde Los diarios de Emilio Renzi no solo vinculan la vida con la literatura, como resulta más evidente, sino la literatura con la vida, en esa suerte de reversibilidad que le ha sido siempre tan cara a Piglia (Laera, 2002). Ahí aparecen, precisamente, las consideraciones retrospectivas sobre el pasado y los recursos utilizados para la publicación de los diarios: la transformación del yo a lo largo del tiempo, el desdoblamiento del autor y el trabajo de edición como modo de reconexión entre el transcurso del tiempo y la instancia textual. Apenas comenzado el relato del primer volumen, se afirma que "Siempre habrá un hiato insalvable entre el ver y el decir, entre la vida y la literatura" (Piglia, 2015: 22). Sobre ese hiato, sobre el modo de sostener ese hiato (iy no de salvarlo!) a través de la constitución del "ser escritor" en el orden de la vivencia y de los procedimientos, es que quiero detenerme.

El editor Piglia entabla con el escritor Piglia/Renzi una relación paradojal. Por un lado, esa figura es imprescindible para que la relación entre vida y literatura se confirme, ya que sería imposible publicar la transcripción de los más de doscientos cuadernos solo por una cuestión de cantidad. Por otro lado, esa figura que hace efectiva la relación es la misma que sostiene el hiato, porque ese editor del diario, el que interviene en los cuadernos, o sea en la escritura y composición de un tipo de autobiografía cuya condición es la cronología, ese editor se instala en ese hiato entre vida y literatura que anuncia el narrador al comienzo para sostenerlo, no para suturarlo. Y lo hace, propongo, conspirando en el presente contra el escritor de los cuadernos del pasado. Vuelvo brevemente a la reseña de Pauls (2016): "los cortes 
-señala- no pueden ser más significativos; prueban, una vez más -dice él-, hasta qué punto las intervenciones del Piglia 'editor', que relee y monta sus diarios décadas después de escribirlos, son tan decisivas, y tan extraordinariamente literarias, como el texto mismo que edita". No quiero detenerme ahora, sin embargo, en el gesto literario de Piglia en tanto editor, porque la operación literaria es quizás lo más evidente (¿no es acaso Piglia un escritor, un escritor ya y para siempre indiscutido a raíz sobre todo de la salida de sus diarios?, ¿no es, aun antes de empezar, una intervención literaria la publicación misma de esos diarios)? Para decirlo en los términos de la poética del cuento que ha expuesto en diversas oportunidades el propio Piglia: ${ }^{4}$ no me interesa acá la historia aparente sino la otra, soterrada, esa segunda historia (de la escritura), ya que en ella puede leerse, además de una puesta al día literaria de los diarios, una teoría conspirativa en el trabajo del editor que apuntala el hiato entre vida y literatura. Me refiero a los alcances de ese índice de ficcionalización que se lee desde el título, Los diarios de Emilio Renzi, y que afecta tanto a la naturaleza de los diarios y a la condición del escritor como a la noción de tiempo que comparten la escritura autobiográfica y la vida de ese escritor.

En líneas generales, hay un procedimiento de edición estratégico y mayor que está en los marcos retroactivos que funcionan como intercalaciones parautobiográficas. En principio, la "Nota del autor" datada el 20 de abril de 2015 , en la que ese "autor" presenta la trilogía recurriendo a un discurso indirecto libre en el que el nombre de Renzi no aparece pero que instala la ambigüedad de la tercera persona hasta su declaración más extrema: "Tiene la extraña sensación de haber vivido dos vidas" (Piglia, 2015: 11). En la misma dirección, están los comienzos de los volúmenes, donde se dan los protocolos de lectura con la presencia de un narrador que parece ser el mismo de la "Nota del autor", quien a través de una conversación entre ambos introduce a Renzi como el escritor al que pertenecen los diarios que da a conocer: "En el umbral" y "En el estudio", que abren respectivamente las dos partes de Años de fornación; "En el bar", que enmarca a Los años felices, y "Sesenta segundos en la realidad", a Un día en la vida. En el primer volumen, además, a estos marcos retroactivos parautobiográficos se les agrega un conjunto de relatos también intercalados, como "El nadador", "Hotel Almagro" o "La moneda griega", entre otros, que en primera o tercera persona corresponden a relatos escritos por esos años y no publicados. En la misma dirección, se encuentra el relato "Un día en la vida", protagonizado por Renzi en tercera persona y narrado en indirecto libre, que está en la mitad del tercer volumen, justo cuando terminan los diarios fechados cronológicamente y antes de los diversos grupos de entradas que llevan su correspondiente título y que se reúnen bajo el título más amplio de, precisamente, "Días sin fecha".

Además de la previsible tarea de edición consistente en seleccionar y corregir los contenidos de los diarios escritos en los cuadernos, el editor realiza una tarea suplementaria que radica en el procedimiento de ficcionalización por el cual se socava la verdad autobiográfica. Así, en los diarios, el editor resulta el suplemento del escritor. El editor, desde esta perspectiva que propongo, no solo edita al escritor, sino que conspira contra él. Y lo hace por una vía doble y hasta paradójica, porque mientras el trabajo de selección sobre los diarios tiene efectos irrecuperables sobre la vida narrada (no se puede saber qué es lo que quedó afuera de los diarios o lo que se agregó), el procedimiento de ficcionalización enfatiza la manipulación y es totalmente autoevidente (el título y los prólogos lo delatan). Sin embargo, el procedimiento más extremo de la conspiración no es ni necesario o previsible ni autoevidente, sino totalmente clandestino. No es parte de la estrategia mayor sino que es una táctica conspirativa, un procedimiento menor y aislado que apenas deja huella, pero que termina de horadar el hiato entre la literatura y la vida porque manipula los tiempos del enunciado y de la enunciación trazando temporalidades alternativas. No se trata de indicar dos temporalidades obvias, por lo tanto, sino de conspirar contra ellas; la
4. En su "Tesis sobre el cuento", Piglia empieza afirmando que un cuento siempre cuenta dos historias, y en la segunda tesis explica que "El cuento clásico (Poe, Quiroga) narra en primer plano la historia 1 (el relato del juego) y construye en secreto la historia 2 (el relato del suicidio). El arte del cuentista consiste en saber cifrar la historia 2 en los intersticios de la historia 1. Un relato visible esconde un relato secreto, narrado de un modo elíptico y fragmentario. El efecto de sorpresa se produce cuando el final de la historia secreta aparece en la superficie." (Piglia, 1999). 
5. Para el modo en que la conspiración trama temas, funcionamientos y procedimientos no solo en la obra de Piglia sino en su modo de pensar el mundo, véase Piglia (2007).

6. Esto habría que leerlo en sintonía con una relación sobre la que Piglia vuelve con insistencia, por ejemplo en un texto de 1987 incluido en Crítica y ficción, libro que recopila ensayos y entrevistas en los que despliega su poética: "no se trata de ver la presencia de la realidad en la ficción (realismo), sino la presencia de la ficción en la realidad (utopía)" (Piglia, 2000). táctica conspirativa corroe, confunde, camufla, simula, desvía, ambas temporalidades, o bien entre sí o bien como si fueran solo una. ¿No está acaso Piglia con el Dipi Di Paola en 1961 cuando escucha "Love me do", el primer single de los Beatles, que fue grabado en 1963 (2015: 115)? ¿No leyó Piglia acaso, como lo menciona en un listado de escritores de comienzos de los 60, a Paul Auster, aun antes de que este escribiera su primera novela? ¿Son acaso lapsus, problemas de una memoria, la del escritor editor, ya mayor, ya enfermo, que interviene en la experiencia sin poder recuperarla por completo? ¿O son conspiraciones de ese editor que ya no es el mismo escritor del que narra su "ser escritor", como propongo pensar? Tácticas de intervención que dejan huellas, marcas que impulsan a buscar fanáticamente la conspiración, a reconstruir esa segunda historia, oculta, clandestina, que lo altera todo; que incitan a descubrir las constantes en esas huellas que parecen azarosas, como ocurría en "La loca y el relato del crimen", por ejemplo (Piglia, 1994).

Estamos ante un editor que, lejos de practicar suturas con sus intervenciones, lo que hace es sostener el hiato entre vida y literatura anunciado desde el comienzo. Si la importancia temática y conceptual de la conspiración servía para intentar unir discursivamente historia y ficción en Respiración artificial y realidad y ficción en La ciudad ausente, o si servía para hacer reversible esa misma relación en Plata quemada, si intentaba ser sistematizada en diversos niveles de la novela en Blanco nocturno para serlo finalmente por completo en El camino de Ida, acá, en Los diarios de Emilio Renzi, todo eso se vive en primera persona. ${ }^{5}$ Esas intervenciones clandestinas que conspiran contra la vida (del escritor y de su relato cronológico) le dan, paradójicamente, un estatuto ficcional a la vida que, en lugar de alejarla de la vida misma para acercarla a la literatura, trabajan en ese hiato irreductible entre ambas. ${ }^{6}$

Así entonces, este editor conspira contra la escritura autobiográfica del escritor. Pero, ¿cómo saber exactamente quién es quién? El editor, hay que decirlo, no es ya ni el escritor que se siente escritor, ni el escritor que escribe, ni el que es escritor. No es más, quiero enfatizarlo, el que ha escrito los diarios. El escritor editor, el nuevo escritor, tiene que ser, él también, el último escritor.

\section{Los diarios y los tiempos}

En una nota para la prensa que me pidieron a propósito de la muerte de Piglia a comienzos de 2017, o sea antes de la salida del último volumen, me referí a los diarios como postestamento. En ellos, escribí, Piglia da cuenta de Todo. Transcribo el final de ese texto:

Los Diarios muestran una trama exacta entre la literatura, la escritura y la vida que, antes de ellos, no había asumido por completo su forma. Porque la intervención de Piglia, ya enfermo, ya de vuelta de su literatura, en sus cuadernos autobiográficos, no es corrección, no es estilismo, sino procedimiento. Es en esa hibridación-del pasado y el presente, en su apuesta al futuro- donde nos lega la cifra para volver a leerlo. (...) Y con ese acto de escritor pródigo, Piglia se reinventa, y nos reinventa así otra relación posible, deseada, entre la literatura y la vida. (Laera, 2017)

Agrego ahora: son reinvenciones fuera de todo tiempo, en un tiempo vacío y heterocrónico que conspira contra las ideas cronológicas, secuenciales, causales, progresivas que todavía reinan en nuestra comprensión de lo que acontece. Como si ser escritor finalmente fuera, una vez que se asume esa condición, conspirar contra ella.

A mediados de 2017, tras la muerte de Piglia a comienzos de ese año, se conoce finalmente el último volumen de Los diarios de Emilio Renzi, cuyo título es Un día en 
la vida. Pasando la mitad, según ya dije, hay un relato protagonizado por Renzi en tercera persona, también llamado "Un día en la vida", que separa los diarios fechados de varios grupos de entradas sin fecha. Se trata de una especie de parábola que responde a un sueño del protagonista. Renzi sueña que en un futuro muy lejano en la costa de Quequén, un pueblo de la provincia de Buenos Aires, aparece un antiguo ejemplar de los diarios: dos pescadores de un buque chino lo encuentran y lo dejan abandonado en la arena por desconocer su posible valor, hasta que por la tarde el libro es recuperado y pasa a formar parte de una exigua Biblioteca Nacional. Los investigadores de ese entonces, quienes hacen crítica filológica (en la Universidad de Buenos Aires) y crítica historicista (en la Universidad de La Plata), discuten si el libro, del que no se pueden obtener los datos por su deterioro, corresponde a comienzos del siglo XXII o a la fecha entre 1957 y 2017 que presentan las entradas que lo componen. También desconocen, si es que lo tiene, el nombre del autor, o si se trata solo de un escribiente. Como sea, en ese mundo del futuro, del que se dirá que Renzi después llamaría "tiempo axial", ya no hay libros ni impresos ni autores tal como los conocemos en la actualidad: la revolución digital terminó con todo eso, a lo que hay que sumarle una posterior catástrofe que, según puede sospecharse, fue total. El mundo es otro y los rasgos de la cultura literaria, los rasgos de esto mismo de lo que estamos hablando, son apenas huellas que buscan reconstruirse pero no repetirse. Por supuesto, y sobre todo si atendemos a los ecos del Borges de "Tlön, Uqbar, Orbis Tertius", el relato es casi ingenuo o previsible: ni siquiera es la invención de un mundo sino que es un sueño. Pero no deja por eso mismo de ser interesante que aparezca ahí, que la manera de instalar un umbral entre el diario fechado y las entradas agrupadas sin fecha sea un relato de imaginación que consiste, justamente, en un sueño, es decir en una suerte de escritor imaginado del mundo futuro para ese libro que se está escribiendo. Si en el presente el escritor es Piglia y es Renzi a la vez, si el escritor del presente conspira ficcionalizadamente en tanto editor contra el escritor del pasado a través del desdoblamiento, el escritor del futuro ya está por completo desligado de toda autoría: ha perdido su nombre. La vida narrada en los diarios, a esa altura, ya no le pertenece a nadie, no encarna en un cuerpo. Sin autor ni editor ni escritor, apenas se reconoce un escribiente copiando un texto que no es más ni del orden de la vida ni de la literatura. El hiato entre vida y literatura, en el sueño de la cultura literaria del futuro, es pura conspiración del tiempo.

Ese relato, propongo, es también un postestamento, una cifra. A partir de allí, las entradas sin fecha se agrupan bajo títulos. Piglia a esa altura ya está enfermo, su cuerpo va perdiendo gradualmente movilidad. El escritor, como el del sueño, va perdiendo sus atributos a la vez que va perdiendo su vida. El capítulo que cierra los "Días sin fecha" se llama "La caída". Allí Piglia/Renzi dicen, de otro modo, desde otra perspectiva, lo mismo que al comienzo de los diarios se expresaba como lanzamiento a futuro: "Siempre quise ser el hombre que escribe." (Piglia, 2017: 294). Pero, ¿cómo es ahora, material y simbólicamente, ese escritor? Cómo es cuando va perdiendo sus atributos materiales: deja de usar una mano, después la otra, después solo puede grabar lo que escribe... Cómo es cuando, y de allí lo postestamentario, resulta irrecuperable porque la materialidad del escribiente, aquel mismo en el que se convertía el autor en el sueño, es precisamente lo que se ha perdido. Cómo es cuando solo queda la dimensión imaginaria del autor que ya no puede ser un escritor: "El genio es la invalidez." (2017: 294). Esa es la última frase de Los diarios de Emilio Renzi de Ricardo Piglia. Así cierra Piglia, con la voz de Renzi y con su cuerpo inmóvil al borde de la muerte, el postestamento de la vida de escritor lanzada al futuro de la literatura. El hiato no podría ser mayor. 


\section{Q Bibliografía}

»Borges, J. L., Ferrari, O. (2005). En diálogo / 1. México: Siglo XXI Editores.

"De Man, P. (1979). “Autobiography by De-facement”. En MLN, vol. 94, núm. 5, Comparative Literature, pp. 919-930.

» Gusdorf, G. (1991 [1948]). “Condiciones y límites de la autobiografía”. En Anthropos. La autobiografía y sus problemas teóricos. Estudios e investigación documental, núm. 29, pp. 9-18.

» Heinich, N. (200o). Être écrivain. Création et identité. Paris : Éditions La Découverte.

»Laera, A. (2002). “Piglia-Eloy Martínez. Contribuciones a la relación entre realidad y ficción en la literatura argentina”. En Milpalabras (letras y artes en revista), núm. 3, 25-30.

"Laera, A. (2017). "Ricardo Piglia, un escritor que aunó tradición y vanguardia”. En La Capital, Rosario, 15 de enero.

»Lahire, B. (2006). La condition littéraire. La double vie des écrivains. Paris : La Découverte.

» Lejeune, P. (1975). Le pacte autobiographique. Paris : Seuil.

»Pauls, A. (2016). “El libro de la semana, por Alan Pauls, Los diarios de Emilio Renzi: Los años felices, de Ricardo Piglia”. En Telam, 28 de octubre.

»Payró, R. J. (1984). Obras. Caracas: Biblioteca Ayacucho.

»Payró, R. J. (1907). El triunfo de los otros. Buenos Aires: M. Rodríguez Giles.

»Piglia, R. (1993). “Echeverría y el lugar de la ficción”. En La Argentina en pedazos. Buenos Aires: Ediciones de la Urraca.

»Piglia, R. (1994). “La loca y el relato del crimen”. En Nombre falso. Buenos Aires: Seix Barral, pp. 65-72

»Piglia, R. (1999). “Tesis sobre el cuento”. En Formas breves. Buenos Aires: Temas Grupo Editorial.

"Piglia, R. (2000). Crítica y ficción. Buenos Aires: Seix Barral.

»Piglia, R. (2007). Teoría del complot. Buenos Aires: Mate.

» Piglia, R. (2015). Los diarios de Emilio Renzi. Años de formación. Barcelona: Anagrama.

»Piglia, R. (2016). Los diarios de Emilio Renzi. Los años felices. Barcelona: Anagrama.

» Piglia, R. (2017). Los diarios de Emilio Renzi. Un día en la vida. Barcelona: Anagrama.

» Sarmiento, D. F. (1979 [1850]). Recuerdos de provincia. Buenos Aires: Centro Editor de América Latina.

»Starobinski, J. (1970). “Le style de l'autobiographie”. En Poétique, núm. 3, pp. 70-89. 